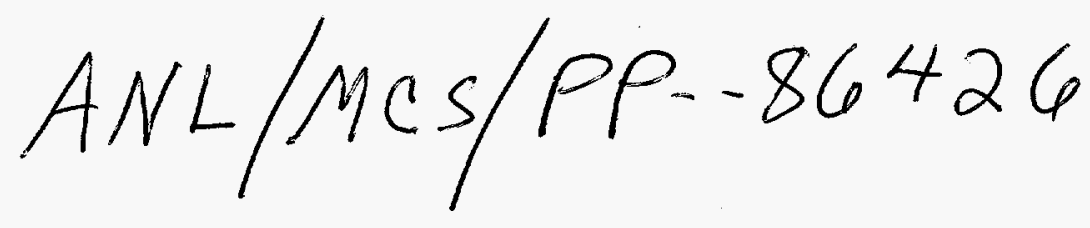

\title{
Centrifugal Destabilization and Restabilization of Plane Shear Flows
}

\author{
A. J. Conley \\ Mathematics and Computer Science Division \\ Argonne National Laboratory \\ Argonne, IL 60439
}

\begin{abstract}
The flow of an incompressible viscous fiuid between parallel plates becomes unstable when the plates are tumbled. As the tumbling rate increases, the flow restabilizes. This phenomenon is elucidated by pathfollowing techniques. The solution of the Navier-Stokes equations is approximated by spectral techniques. The linear stability of these solutions is studied.
\end{abstract}

\section{Introduction}

Recently, studies of plane Couette flow in the presence of a Coriolis force have led to the discovery of new solutions of the Navier-Stokes equations (see [Nag90] [Con94] [CK]). One observation of these studies has been that, as the Coriolis force is increased, the parallel shear flow bifurcates to a new solution that reconnects to the parallel flow at larger Coriolis force. This paper is a description of similar phenomena in Poiseuille flow.

Poiseuille flow is the inviscid, incompressible flow between infinite, parallel, stationary plates (see Fig. 1). The addition of a Coriolis force causes plane Poiseuille flow to become unstable. The solutions that bifurcate from plane Poiseuille flow are stable and, at larger Coriolis force, reconnect to plane Poiseuille flow. Thus the Coriolis force destabilizes the flow and restabilizes it.

Define

$$
\vec{U}(x, y, z)=\left(\begin{array}{c}
U(x, y, z) \\
V(x, y, z) \\
W(x, y, z)
\end{array}\right)
$$

to be the velocity of the flow at location $(x, y, z)$ (see Fig. 1). Define $P(x, y, z)$ to be the pressure of the flow. Traveling wave solutions of the Navier-Stokes 


\section{DISCLAMIER}

Portions of this document may be illegible in electronic image products. Images are produced from the best available original document. 


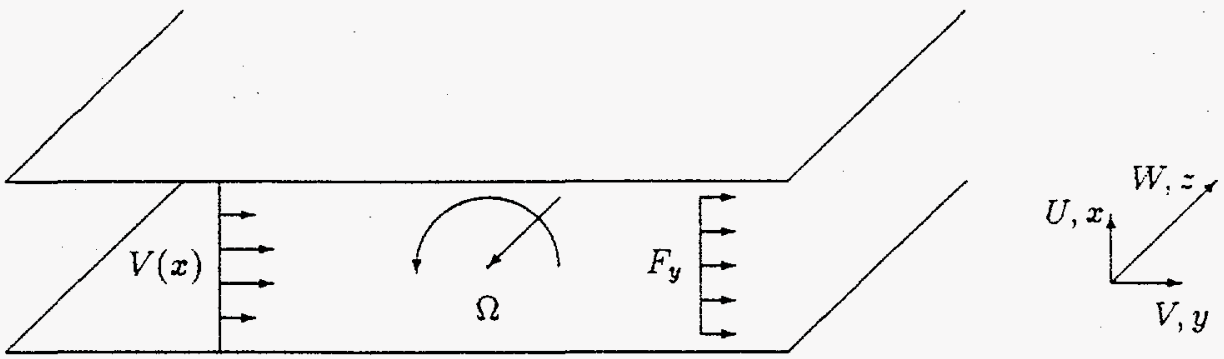

Figure 1: The flow of an incompressible viscous fluid between parallel infinite plates subject to a pressure gradient is called Poiseuille flow. The plates are tumbling with angular velocity, $\Omega$.

equations for flow between parallel, tumbling plates satisfy the following:

$$
\begin{aligned}
\frac{\partial \vec{U}}{\partial t}= & -\operatorname{Re}(\vec{U} \cdot \vec{\nabla}) \vec{U}+\nabla^{2} \vec{U}-\vec{\nabla} P+2 \Omega(\tilde{z} \times \vec{U}) \\
& +c_{y} \partial_{y} \vec{U}+c_{z} \partial_{z} \vec{U}+F_{y} \hat{y}, \\
0= & \vec{\nabla} \cdot \vec{U} .
\end{aligned}
$$

Here $c_{y}$ and $c_{z}$ are the wave speeds in the $y$ and $z$ directions, respectively; $F_{y}$ is the force on the fluid in the $y$ direction; and $\Omega$ is the tumbling rate of the plates. The solutions of these equations are required to satisfy no-slip boundary conditions at the plates $(x= \pm 1 / 2)$ and periodic boundary conditions in the $y$ and $z$ directions:

$$
\begin{aligned}
\vec{U}\left( \pm \frac{1}{2}, y, z\right) & =0, \\
\vec{U}\left(x, y+\lambda_{y}, z\right) & =\vec{U}(x, y, z), \\
P\left(x, y+\lambda_{y}, z\right) & =P(x, y, z), \\
\vec{U}\left(x, y, z+\lambda_{z}\right) & =\vec{U}(x, y, z), \\
P\left(x, y, z+\lambda_{z}\right) & =P(x, y, z) .
\end{aligned}
$$

To graph the flows, we introduce the wall-shear force,

$$
\tau(\vec{U})=\frac{1}{\lambda_{y} \lambda_{z}} \int_{0}^{\lambda_{y}} d y \int_{0}^{\lambda_{z}} d z \frac{d V}{d x}(1 / 2, y, z)
$$

Plane Poiseuille flow,

$$
\vec{U}(x, y, z)=\left(\begin{array}{c}
0 \\
F_{y} / 2\left(1 / 4-x^{2}\right) \\
0
\end{array}\right),
$$


satisfies these equations for all $\Omega$. This paper describes solutions that bifurcate from plane Poiseuille flow as $\Omega$ is varied. Plane Poiseuille flow refers to the solution (1.9) above; Poiseuille flow refers to any solution of Eqs. (1.1)-(1.7).

Section 2 describes the approximation. Section 3 describes the path-following methods and Section 4 the eigenvalue paths. Section 5 contains the results of this formulation, and Section 6 discusses the results.

\section{Approximation of Velocity and Pressure Fields}

The solutions are expanded in terms of

$$
\begin{gathered}
C_{l, m, n}(x, y, z)=T_{l}(2 x) \cos \left(m \alpha_{y} y+n \alpha_{z} z\right), \\
S_{l, m, n}(x, y, z)=T_{l}(2 x) \sin \left(m \alpha_{y} y+n \alpha_{z} z\right), \\
A_{l, m, n}=\left(C_{l, m, n}+i S_{l, m, n}\right)=T_{l}(2 x) e^{i\left(m \alpha_{y} y+n \alpha_{z} z\right)} .
\end{gathered}
$$

Here, $\alpha_{y}=\frac{2 \pi}{\lambda_{y}}$ and $\alpha_{z}=\frac{2 \pi}{\lambda_{z}}$ are the $y$ and $z$ wave numbers, respectively. Each of $U, V, W$, and $P$ is approximated by finite expansions of the form

$$
q(x, y, z)=\sum_{l=0}^{L} \sum_{m=-M}^{M} \sum_{n=-N}^{N} q_{l, m, n} A_{l, m, n}(x, y, z) .
$$

As a result, the approximated $\vec{U}$ and $P$ are determined by a total of $4(L+$ 1) $(2 M+1)(2 N+1)$ coefficients. The same notation for the approximations and the solutions is used in this paper.

These approximations do not (in general) satisfy the Navier-Stokes equations. As a result, only certain projections of the Navier-Stokes equations are required to be zero. Define the inner products,

$$
\begin{aligned}
\left\langle A_{l m n}, f\right\rangle & =\int_{1 / 2}^{1 / 2} \frac{d x}{\sqrt{1-4 x^{2}}} \int_{0}^{\lambda_{y}} d y \int_{0}^{\lambda_{z}} d z\left(A_{l m n} \cdot f\right), \\
\left\langle e^{i\left(m \alpha_{y} y+n \alpha_{z} z\right)}, f\right\rangle & =\int_{0}^{\lambda_{y}} d y \int_{0}^{\lambda_{z}} d z\left(e^{i\left(m \alpha_{y} y+n \alpha_{z} z\right)} f\right) .
\end{aligned}
$$

Define $\vec{M}$ to be the right-hand side of Eq. (1.1). Then the approximations are required to satisfy the following $4(L+1)(2 M+1)(2 N+1)$ equations:

$$
\begin{aligned}
\left.<A_{l m n}, \vec{M}\right\rangle=0\left\{\begin{array}{l}
0 \leq l \leq L-2, \\
|m| \leq M, \\
|n| \leq N
\end{array}\right. \\
<A_{l m n}, \vec{\nabla} \cdot \vec{U}>=0\left\{\begin{array}{l}
0 \leq l \leq L-2, \\
|m| \leq M, \\
|n| \leq N
\end{array}\right. \\
\left\langle e^{i\left(m \alpha_{y} y+n \alpha_{z} z\right)}, \vec{U}( \pm 1 / 2, y, z)\right\rangle=0\left\{\begin{array}{l}
|m| \leq M, \\
|n| \leq N .
\end{array}\right.
\end{aligned}
$$


Equations (2.12)-(2.14) are referred to as

$$
F(u, \lambda)=0
$$

in the rest of this paper. The solution, $u$, is the set of coefficients that determine $\vec{U}$ and $P$. The parameter, $\lambda$, is typically $\Omega, R e$, or $\alpha_{z}$.

Since the vortical flows have a phase freedom in $z$, the following phase constraint is imposed on the solutions:

$$
\Phi_{z}(u)=<C_{001}, U(x, y, z)>=0 .
$$

On the plane Poiseuille branch of solutions, the phase constraint is trivially satisfied, and the wave speed, $c_{z}$, is undefined. When the phase constraint is imposed (on the vortical flow), it is adjoined to $F(u, \lambda)$ and $c_{z}$ is solved for $u$ (in addition to the coefficients).

\section{Path Following}

Continuation methods are used to approximate the solution path:

$$
\Gamma=\{(u, \lambda): F(u, \lambda)=0\} .
$$

Distinct methods are employed to follow regular paths and to switch paths of solutions. In the case of regular path segments, the following algorithm is used:

Step 1. Start with an initial solution, $\left(u_{0}, \lambda_{0}\right)$. Construct the Jacobian,

$$
F_{u}^{0}=\frac{\partial F\left(u_{0} ; \lambda_{0}\right)}{\partial u}
$$

Step 2. Construct the initial iterate and initial parameter with one of the following:

- constant value continuation: $\lambda_{i}=\lambda_{i-1}+\delta \lambda, u_{i}^{0}=u_{i-1}$, or

- secant continuation: $\lambda_{i}=\lambda_{i-1}+\delta \lambda$, $u_{i}^{0}=u_{i-1}+\frac{\lambda_{i}-\lambda_{i-1}}{\lambda_{i-1}-\lambda_{i-2}}\left(u_{i-1}-u_{i-2}\right)$.

Step 3. Compute the special Newton iterates (indexed by $\nu=1,2, \ldots$ ),

$$
\begin{gathered}
F_{u}^{0} \epsilon^{\nu}=-F\left(u_{i}^{\nu} ; \lambda_{i}\right), \\
u_{i}^{\nu+1}=u_{i}^{\nu}+\epsilon_{i}^{\nu},
\end{gathered}
$$

until $\left\|\epsilon_{i}^{N}\right\|_{l_{1}}<\epsilon$.

Step 4. Set $u_{i}=u_{i}^{N+1}$. If the parameter $\lambda_{i}$ is still in the desired range and the number of iterations is small (e.g., $N<30$ ), return to Step 2. 
This algorithm may fail for several different reasons. One is that the step $\delta \lambda$ is too large. In this case, decrease the step size. Another reason the algorithm may fail is that the iterates in Step 3 converge too slowly. In this case recompute the Jacobian. Lastly, in the case that the solution path has trespassed a bifurcation point, switch paths as described later in this section, or predict a solution on the opposite side of the bifurcation point and continue with Step 3 of the algorithm above.

To switch from plane Poiseullle flow to the vortical flow solution, use the following algorithm:

Step 1. Accurately find the parameter value $\lambda^{*}$ at which

$$
F_{u}^{*}=\frac{\partial F\left(u\left(\lambda^{*}\right), \lambda^{*}\right)}{\partial u}
$$

is singular.

Step 2. Find the right null vector, $\phi$, of $F_{u}^{*}$ satisfying $\Phi_{z}(\phi)=0$.

Step 3. Construct the initial guess for a solution on the new branch, $u_{i}^{0}=$ $u\left(\lambda^{*}\right)+\epsilon \phi$.

Step 4. Compute the Newton iterates for the augmented system,

$$
\left(\begin{array}{c}
F\left(u, c_{z}, \lambda\right) \\
\phi \cdot u \\
\Phi_{z}(u)
\end{array}\right)=\left(\begin{array}{c}
0 \\
\phi \cdot u_{i}^{0} \\
0
\end{array}\right) .
$$

The parameters $c_{z}$ and $\Omega$ have been freed as variables.

The path of vortical flow is regular if the phase constraint is adjoined to $F(u, \lambda)$ and $c_{z}$ is solved for (in addition to $u$ ). As a result, the algorithm for a regular path can be used, but the augmented system of equations,

$$
H\left(u, c_{z}, \lambda\right)=\left(\begin{array}{c}
F\left(u, c_{z}, \lambda\right) \\
\Phi_{z}(u)
\end{array}\right)=0
$$

should be used instead of $F(u, \lambda)=0$.

\section{Eigenvalue Paths}

Once a path of solutions has been computed, it is useful to know the stability of these solutions. The stability is given by the eigenvalues, $\mu$, of the linear system,

$$
\begin{aligned}
\mu \vec{\epsilon}= & -\operatorname{Re}\left(\vec{U}_{0} \cdot \vec{\nabla}\right) \vec{\epsilon}-\operatorname{Re}(\vec{\epsilon} \cdot \vec{\nabla}) \vec{U}_{0}+\nabla^{2} \vec{\epsilon}-\vec{\nabla} \rho+2 \Omega(\hat{z} \times \vec{\epsilon}) \\
& +c_{y} \partial_{y} \vec{\epsilon}+c_{z} \partial_{z} \vec{\epsilon} \\
0= & \vec{\nabla} \cdot \vec{\epsilon}
\end{aligned}
$$




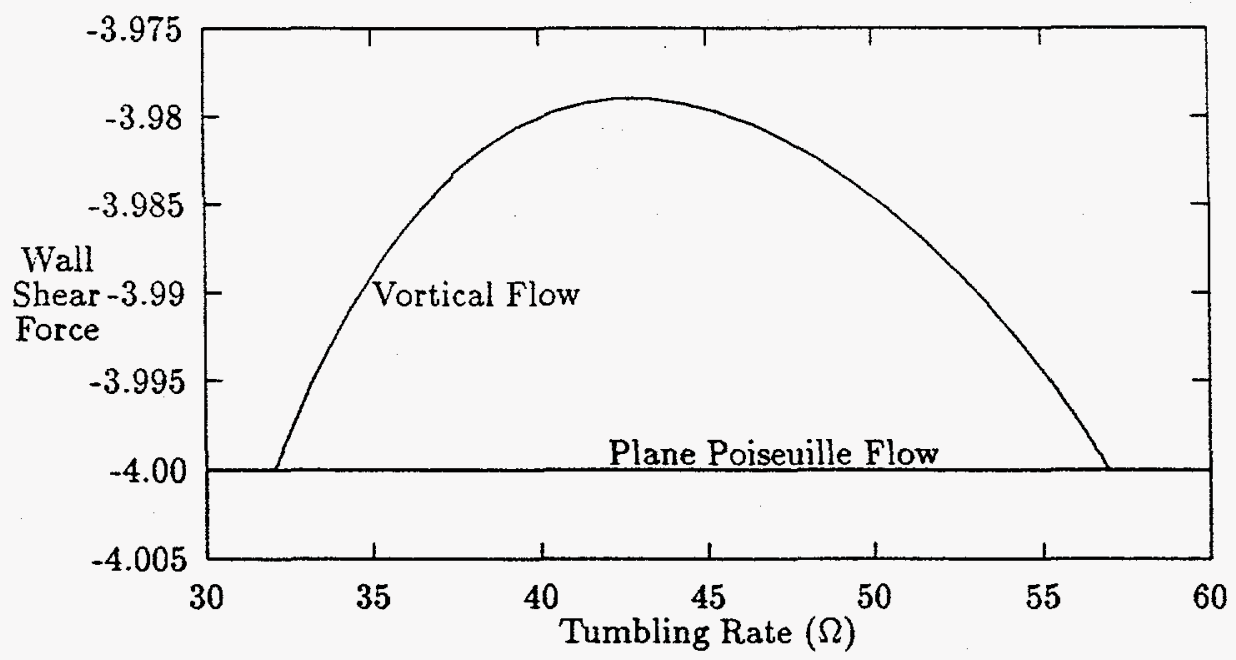

Figure 2: A path of vortical flows bifurcates from plane Poiseuille flow and then reconnects at larger tumbling rates. $R e=140, \alpha_{z}=4.0, F_{y}=8, c_{z}=0, L=$ $14, N=9$.

The above system is obtained from Eqs. (1.1)-(1.7) by letting $\vec{U}=\vec{U}_{0}(\lambda)+$ $\bar{\epsilon}(\lambda) e^{\mu t}, P=P_{0}(\lambda)+\rho(\lambda) e^{\mu t}$, and ignoring terms of order $\left(\epsilon^{2}\right) . \vec{U}_{0}$ is a solution of Eqs. (1.1)-(1.7) with $\frac{\partial \vec{U}_{0}}{\partial t}=0$. As the solution $\vec{U}_{0}$ varies with the parameter $\lambda$, the eigenvalues of the above equations, $\mu$, vary. This variation leads to paths of eigenvalues, $\mu(\lambda)$, which are functions not only of $\lambda$ but also the particular path of solutions $\vec{U}_{0}(\lambda)$. If Real $(\mu(\lambda))<0$ for all the eigenvalues, then the solution is stable on that path segment. In this paper, $\vec{\epsilon}$ is approximated in the same way as $\vec{U}_{0}$ with the same periodicities and resolution.

\section{$5 \quad$ Results}

For a fixed Reynolds number, the critical tumbling rate (i.e., the value of $\Omega$ at which vortical flow bifurcates from plane Poiseuille flow) varies with $\alpha_{z}$ (see Fig. 3). The critical $\Omega$ is minimized when $\alpha_{z}$ is near 4. For a fixed wave number, the relation between Reynolds number and critical $\Omega$ is shown in Fig. 4. 


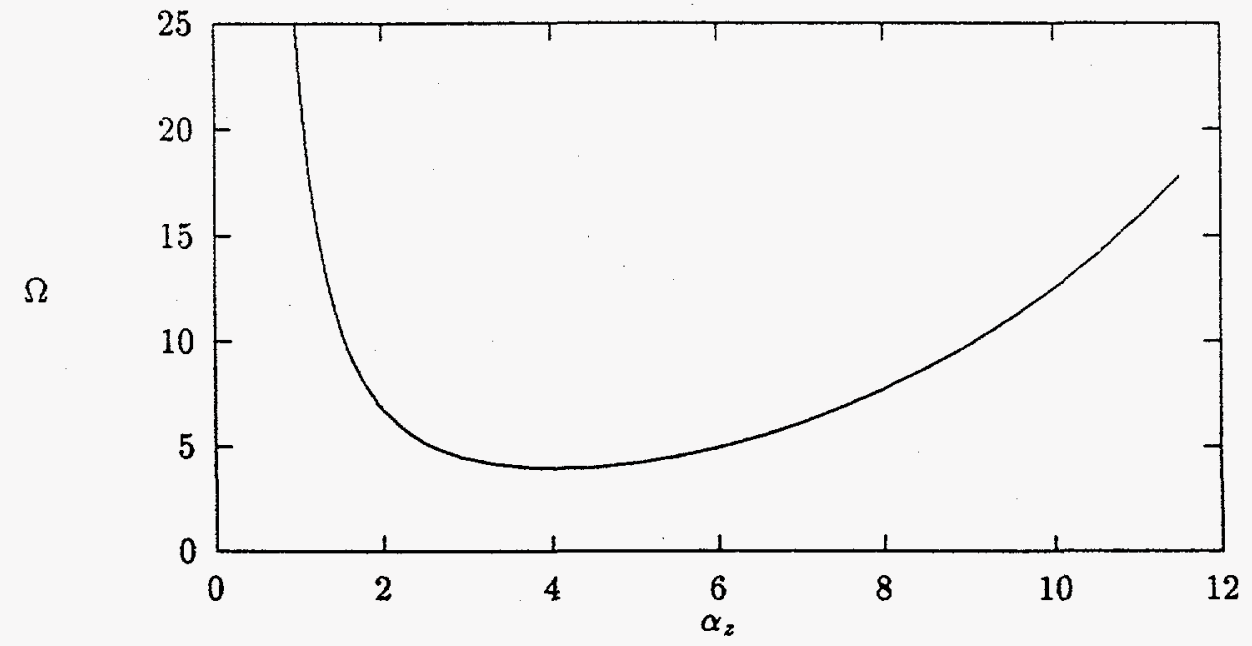

Figure 3: The critical $\Omega$ as a function of $\alpha_{z} . R e=600, L=32, F_{y}=8$.

The branch of vortical flow intersects the branch of plane Poiseuille flow at $R e=140, \Omega_{c}=32.05, \alpha_{z}=4.0$ and again at the larger tumbling rate, $\Omega_{\mathrm{e}}=56.95$, as can be seen in Fig. 2.

Linear stability analysis yields the paths of eigenvalues seen in Fig. 5. The three branches of eigenvalues associated with the linear stability of plane Poiseuille flow in Fig. 5 are double eigenvalues. As can be seen from the figure, plane Poiseuille flow is unstable when the tumbling rate is in the range between the two bifurcation points. In contrast, the vortical flow is stable along its full path. (The zero eigenvalue is due to the phase freedom of the solution in the $z$ direction.)

\section{Discussion}

While Eqs. (1.1)-(1.7) (with $F_{y}=8, \Omega=0$ ) are known to have traveling wave solutions for large Reynolds numbers (see [DR81]), these flows vary with $x$ and $y$. In contrast, the vortical flow (stationary waves) calculated here vary with $x$ and $z$. The vortical flow is stable (at this Reynolds number and wave number) for all values of $\Omega$ for which it exists. In contrast, plane Poiseuille flow is stable for values of $\Omega$ for which the vortical flow does not exist. At larger Reynolds numbers, this picture may be more complicated as a result of the existence of 


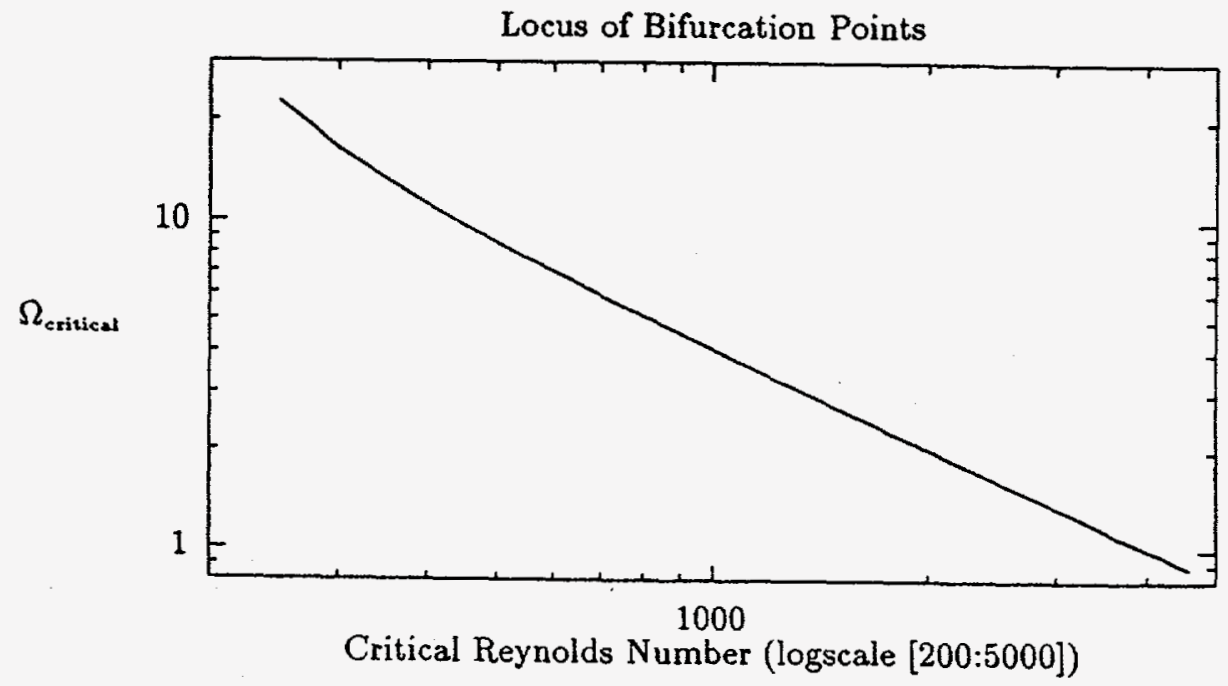

Figure 4: Critical Reynolds number vs critical $\Omega$ with 32 Chebyshev polynomials. $F_{y}=8, \alpha_{z}=1.96$.

\section{DISCLAIMER}

This report was prepared as an account of work sponsored by an agency of the United States Government. Neither the United States Government nor any agency thereof, nor any of their employees, makes any warranty, express or implied, or assumes any legal liability or responsibility for the accuracy, completeness, or usefulness of any information, apparatus, product, or process disclosed, or represents that its use would not infringe privately owned rights. Reference herein to any specific commercial product, process, or service by trade name, trademark, manufacturer, or otherwise does not necessarily constitute or imply its endorsement, recommendation, or favoring by the United States Government or any agency thercof. The views and opinions of authors expressed herein do not necessarily state or reflect those of the United States Government or any agency thereof. 


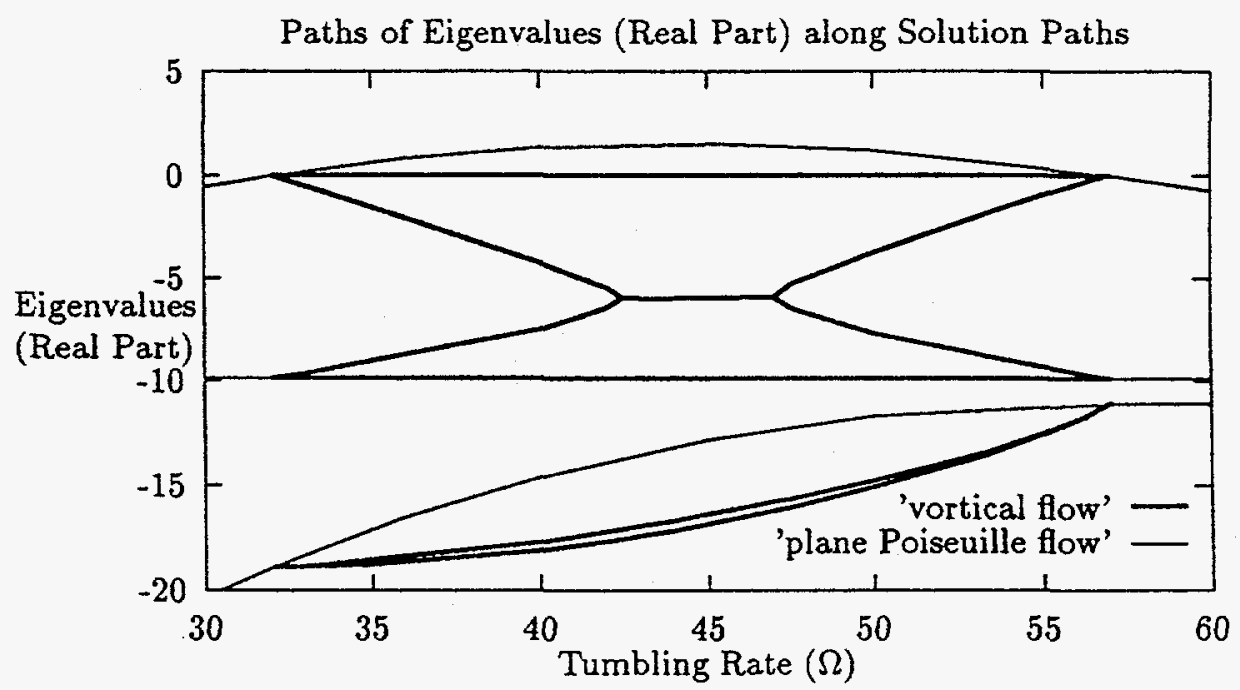

Figure 5: The (real part of) eigenvalues resulting from the linear stability analysis of both plane Poiseuille flow and vortical flow are graphed here as a function of $\Omega$. All three paths of eigenvalues of the plane Poiseuille flow path are double eigenvalues. $F_{y}=8, c_{y}=400, R e=140, \alpha_{y}=2, \alpha_{z}=4, L=14, M=1, N=3$. 


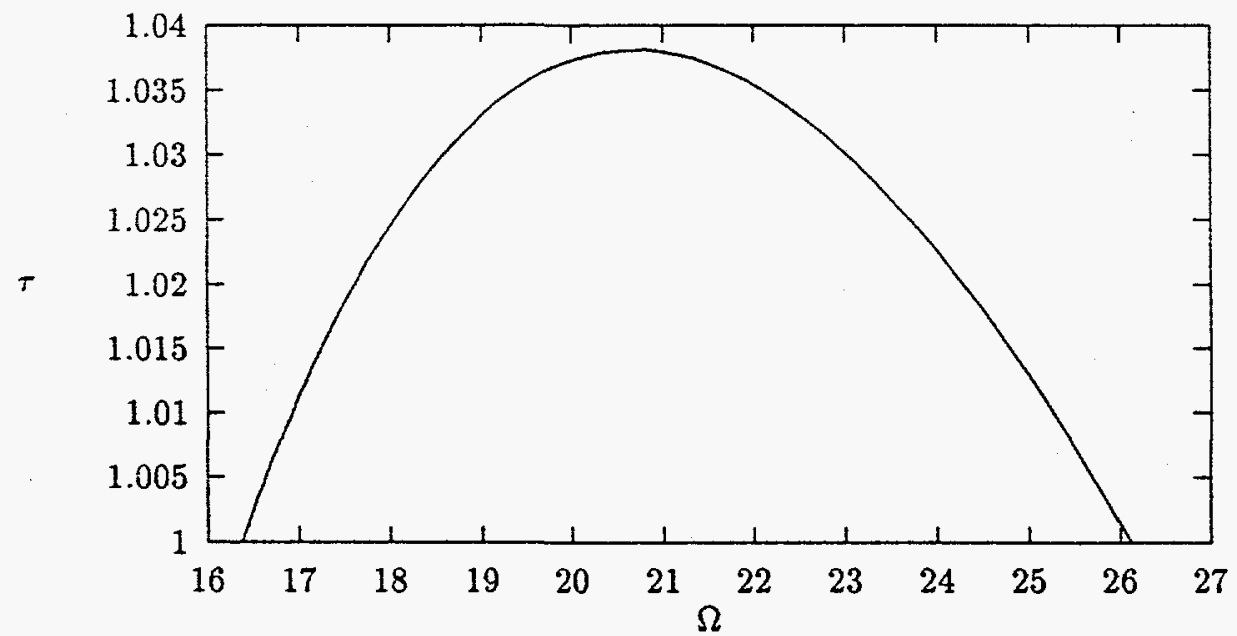

Figure 6: Shear stress vs. $\Omega$ at $R e=85.0, \alpha_{z}=3.0, \alpha_{y}=1.6$. This branch of vortices shows that the two values of $\Omega$ satisfying $2 \Omega(R e-2 \Omega)=T_{c}$ correspond to bifurcation points on the same branch of vortex solutions.

other bifurcating branches of solutions.

A similar phenomenon occurs in Couette flow, as can be seen in Fig. 6; a solution bifurcates from Couette flow and rejoins at larger tumbling rates. These results are obtained by the methods described in [CK]. In Couette flow it is easy to compute successive bifurcations (at larger Reynolds numbers) to threedimensional flows which exist with nontumbling plates (see [CK]). It seems that these solutions should exist in Poiseuille flow but would probably be traveling waves.

This paper has presented analysis of the effect of tumbling plane shear flow. This tumbling rate acts to destabilize plane Poiseuille flow and plane Couette flow. At higher tumbling rates the flows are restabilized. There is a single path of solutions that link the bifurcation points where plane shear flows becomes destabilized and restabilized.

\section{Acknowledgment}

This work was supported by the Office of Scientific Computing, U.S. Department of Energy, under Contract W-31-109-Eng-38. 


\section{References}

[CK] A. J. Conley and H. B. Keller. Wavy Taylor vortices in plane Couette flow. Preprint MCS-P495-0195. Mathematics and Computer Science Division, Argonne National Laboratory, 1995.

[Con94] A. J. Conley. New Plane Shear Flows. PhD thesis, California Institute of Technology, 1994.

[DR81] P. G. Drazin and W. H. Reid. Hydrodynamic Stability. Cambridge University Press, 1981.

[Nag90] M. Nagata. Three-dimensional finite-amplitude solutions in plane Couette flow: Bifurcation from infinity. J. Fluid Mech., 217:229-250, 1990. 